\title{
Large-scale patterns in trematode richness and infection levels in marine crustacean hosts
}

\author{
David W. Thieltges ${ }^{1, *}$, Brian L. Fredensborg ${ }^{2}$, Anja Studer ${ }^{1}$, Robert Poulin ${ }^{1}$ \\ ${ }^{1}$ Department of Zoology, University of Otago, PO Box 56, New Zealand \\ ${ }^{2}$ Department of Biology, University of Texas-Pan American, 1201 W University Drive, Edinburg, Texas 78539, USA
}

\begin{abstract}
Little is known about the patterns of variation in parasitism in marine hosts. Trematodes, the dominant parasites in intertidal systems, are transmitted from their first intermediate hosts (snails) to a range of second intermediate hosts, including crustaceans. Using published studies of trematode infections in crustacean hosts, we investigated general patterns of variation in trematode species richness and infection levels (i.e. percentage of hosts infected and mean number of individual parasites per host). Since the production and release of infective stages in snails is strongly temperature dependent, we also investigated a potential decrease in trematode infection levels with increasing latitude (as a proxy for decreasing temperature). Trematode species richness in the crustacean hosts was generally low (mostly 1 or 2), and infection levels were moderate. However, there were differences among taxa in some groups, particularly among brachyuran crabs, which showed significantly higher values than in other groups. For amphipods, which were the only well-studied group across a large range of latitudes, we found negative correlations between latitude and the trematode species richness or measures of infection level considered here. These relationships persisted after correction of the potentially confounding effects of sampling effort, host body size and host generic identity (as a control for phylogenetic influences). We discuss these findings in light of environmental mediation of parasite transmission, in particular with respect to the probably fundamental role of temperature in driving the output of trematode infective stages in marine systems.
\end{abstract}

KEY WORDS: Parasitism · Trematodes · Metacercariae $\cdot$ Crustaceans $\cdot$ Infection patterns Latitudinal gradient

Resale or republication not permitted without written consent of the publisher

\section{INTRODUCTION}

One of the most fundamental distributional global patterns is the increase in the richness of free-living species from the poles to the equator (Pianka 1966, Rohde 1992, Willig et al. 2003). This pattern is strikingly universal across taxa, habitats, scale and space as revealed by a recent meta-analysis (Hillebrand 2004a). Marine species are no exception, with even stronger and steeper latitudinal gradients than those of freshwater species (Attrill et al. 2001, Hillebrand 2004b). Various mechanisms have been proposed to underlie the observed pattern, but there is no current consensus (Rohde 1992, Willig et al. 2003, Mittelbach et al. 2007). The proposed mechanisms can be grouped into ecological, evolutionary and historical hypotheses (Mittel- bach et al. 2007). While the focus of ecological hypotheses is on ecological mechanisms of species coexistence and the maintenance of diversity, evolutionary hypotheses are based on the rates of diversification, which supposedly vary with latitude (faster rates at low latitudes). Historical hypotheses place emphasis on the older geological and evolutionary history of the tropics compared to the younger pole-ward areas.

Latitudinal gradients in species diversity are not confined to free-living species, but also occur in parasites. Biogeographic patterns of parasites are best known for metazoan parasites of nearshore or shallow-water marine fishes (Rohde 1993, 2002, Poulin \& Morand 2004). In general, the diversity of ectoparasites increases with decreasing latitude (or increasing water 
temperature) (Rohde 1993, 2002, Poulin \& Rohde 1997, Rohde \& Heap 1998). In contrast, no such pattern seems to exist in the endoparasites of marine fish (Rohde \& Heap 1998, Rohde 2002). As with free-living species, there is no consensus on the underlying mechanisms. However, one hypothesis attempting to explain the diversity gradient in ectoparasites is the evolutionary time hypothesis. In warmer waters, species experience higher rates of speciation because of shorter generation times and higher mutation rates, i.e. evolution proceeds at a faster rate (Rohde 1992, 1998, 1999). However, this hypothesis does not account for the obvious differences between ecto- and endoparasites, and Rohde \& Heap (1998) have proposed other biological differences between the 2 types of parasites to account for the observed pattern.

In the studies mentioned above, fish mostly function as definitive hosts for parasites. Most of these parasites have complex life cycles involving one or more intermediate hosts. Our current knowledge on large-scale patterns of parasitism in intermediate hosts is restricted to trematodes in gastropods, which serve as first intermediate hosts for trematode parasites. In the course of an infection, gastropods are often castrated. Replacing the gonadal tissues of the snails, the parasite asexually produces free-living transmission stages, the cercariae. These are shed into the environment to seek and infect a second intermediate host (e.g. bivalves, crustaceans) in which they encyst and develop into metacercariae. When the second intermediate host is consumed by a definitive host (i.e. bird, fish, or mammal), the life cycle has been completed and starts again with the production of eggs that will infect snails once released into the environment (see Lauckner 1980). Comparative studies of trematode richness in gastropods among or within snail host species suggest that there are no or only very weak latitudinal gradients in species richness among snail-trematode systems (Poulin \& Mouritsen 2003, Thieltges et al. 2009a). Furthermore, there is no evidence for latitudinal gradients in infection levels (i.e. prevalence, or percentage of the population that is infected) in snails (Poulin \& Mouritsen 2003). This might simply reflect the fact that there are no such patterns in the final hosts, which are the prime agents of dispersal for most parasites. Alternatively, it suggests that small-scale heterogeneity in local factors may be more important and overrides any potential large-scale effect of latitude (Poulin \& Mouritsen 2003).

While latitudinal gradients have been explored in first intermediate and definitive hosts, they have not been studied in second intermediate invertebrate hosts in trematode life cycles (mainly bivalves or crustaceans). Particularly with respect to infection levels, we might expect a strong effect of latitude (being a proxy for temperature). The production of cercariae (transmission stage infecting second intermediate hosts) in snails and their release into the environment are strongly positively correlated with temperature (Mouritsen 2002, Poulin 2006, Thieltges \& Rick 2006). In extreme cases, a 200-fold increase in cercarial release can result from a $10^{\circ} \mathrm{C}$ increase in temperature, whereas the mean increase over a $10^{\circ} \mathrm{C}$ rise in temperature across a range of snail-trematode systems is about 8-fold (Poulin 2006). This rate of increase is much more substantial than that expected from the usual basic physiological processes of organisms, for which 2- to 3-fold increases in response to a $10^{\circ} \mathrm{C}$ increase in temperature are normally reported (Schmidt-Nielsen 1997, Willmer et al. 2000). Hence, temperature is the crucial factor in the transmission ecology of cercariae by strongly affecting cercarial production and release. Based on this, all else being equal, we should expect higher infection levels in second intermediate hosts in warmer waters at low latitudes than in colder waters at high latitudes. However, there might be confounding variables dampening this pattern. Host size is well known to affect parasite richness and infection levels, with larger hosts supporting more species and a higher abundance of individual parasites (Poulin \& Morand 2004). From a methodological perspective, sampling effort is critical, as sample size (no. of individual hosts dissected) affects the estimation of parasite richness (Walther et al. 1995) and, to a lesser extent, prevalence (Gregory \& Blackburn 1991). Analyses of large-scale patterns in parasite richness and infection levels must take these confounding factors into account.

Other large-scale patterns of parasitism in second intermediate hosts are also largely unexplored. Are all individuals within populations infected? How high is the parasite burden of individual hosts? And are certain host taxonomic groups more affected than others? Data exploring these aspects would be helpful to evaluate the relevance of trematodes for populations of second intermediate hosts as their effect is generally considered to be dependent on the density of parasites (Fredensborg et al. 2004, Thieltges 2006).

The objective of the present study was to investigate general patterns of parasitism in marine second intermediate hosts across populations throughout the globe. The host group for which most data are available in the literature are crustaceans, and we studied variations in trematode richness and infection levels of crustacean hosts at 3 different taxonomic levels: (1) we describe the frequency distribution of trematode species richness and infection levels in crustacean hosts in general, (2) we test for differences in trematode richness and infection levels among various crustacean taxa, and (3) we investigate latitudinal 
gradients in trematode richness and infection levels in the taxon for which most data are available, the amphipods.

\section{MATERIALS AND METHODS}

Data set. We compiled a data set from published studies that we retrieved from online data bases and our own reprint collections. We only included studies on marine crustacean hosts sampled in marine or brackish waters and studies that gave information on sampling effort together with quantitative measures of infection levels. For each parasite species in a host, we recorded prevalence (proportion of infected hosts in the sample), mean intensity (mean number of parasites per host among infected hosts only), and/or mean abundance (mean number of parasites per host across the whole sample). Only rarely were all 3 of these measures reported in the original publications; if possible, we calculated the other measures (abundance $=$ prevalence $\times$ intensity). In cases where data were only available in the form of graphs, we used the free software Engauge (http://digitizer.sourceforge.net/) to extract the required values. For each entry in the data set, we used only a single richness or infection level value, i.e. when the same population was sampled more than once, total species richness and mean infection levels were entered. Only studies investigating at least 15 individual hosts from the same locality were included, in order to achieve a compromise between the level of accuracy of the recorded infection levels and the number of records for which data would be available. For each entry, the latitude of the sampled location was noted, with latitudes from the northern and southern hemispheres combined together. The majority of samples came from the northern hemisphere, and omitting the few southern samples generally gave similar results in subsequent analyses. We also recorded sampling effort (i.e. the number of host individuals examined) for each entry included in the data set. In addition, we recorded the maximum size of the hosts, either as reported in the original publications or in the literature (identification guides, taxonomic keys, etc.).

Analyses. We analysed the data set on 3 different taxonomic levels. First, we used the entire data set to calculate the frequency distribution of trematode species richness and infection levels (prevalence, intensity, abundance) over all host groups and populations. Only studies that reported total parasite inventories (and not reports on single parasite species) were used for the calculation of the trematode richness frequency distribution. We also used the entire data set to investigate the potential relationship between prevalence and the intensity of single-species infections, using linear regression analysis. This was used to test whether individuals in populations where infection is frequent also harbour higher numbers of metacercariae.

Second, we tested for differences in the mean number of trematode species and infection levels (prevalence, intensity, abundance) of metacercarial infections among host taxonomic groups with at least 2 entries, using separate 1-way ANCOVAs, with the number of species and the 3 infection level measures as the dependent variables and sampling effort as a covariate. Again, only studies that reported total parasite inventories were used for the analysis of trematode richness patterns. Trematode richness, intensity and abundance and sampling effort were log-transformed to meet the assumptions of parametric tests.

Third, we searched for latitudinal gradients in parasite richness and infection levels in amphipods, the only host taxon for which the numbers of samples and the range of the latitudinal gradient covered by the data were large enough for such an analysis. We used 4 separate general linear models (GLM) to test for effects of latitude on trematode richness, prevalence, intensity and abundance. To account for the potentially confounding effects of sampling effort and host size, we added both variables as additional predictor variables to the models. Furthermore, we used codes for the different genera to account for phylogenetic effects, i.e. potential overrepresentation of certain genera. The genus code was added as a separate predictor variable to the models (genera with $<3$ entries in the data set were lumped into 'others'). Response variables were either trematode species richness, prevalence, intensity, or abundance. Sampling effort, host size, trematode richness, intensity and abundance were logtransformed to meet the assumptions of parametric tests (visual inspection of predicted values versus residuals).

\section{RESULTS}

\section{The data set}

Our data set included 290 entries from metacercarial infections in marine crustacean hosts. In total, the data set contained 62 species of trematode parasites and 78 species of crustacean hosts, i.e. some parasite and host species were recorded at several localities and some parasite species infected several host species. Sample sizes of individual hosts at each locality ranged widely from 15 to 8800 , with a mean $( \pm \mathrm{SD})$ sample size of 388 \pm 835 ind.. Data on all 3 infection measures (prevalence, intensity, abundance) were not available for all entries, thus reducing the number of samples used for 
the various analyses. Most parasite species belonged to the Microphallidae (216 entries), followed by Opecoelidae (60), unknown taxa (6), Zoogonidae (5), Derogenidae (2) and Brachycoelidae (1). Crustacean groups for which data were available included amphipods (Amphipoda) (130 entries), crabs (Brachyura) (47), isopods (Isopoda) (44), shrimps (Caridea) (27), barnacles (Cirripedia) (25), anomurans (Anomura) (13), prawns (Dendrobranchiata) (3) and lobsters (Palinurida) (1). Data compiled for amphipod hosts covered a latitudinal range from $37^{\circ}$ to $70^{\circ}$ for trematode species richness data, and from $34^{\circ}$ to $55^{\circ}$ for prevalence, intensity and abundance data. From latitudes above and below these ranges, no published data were available. The complete data set is included as supplementary material in the electronic appendix (see Table S1, available at: www.int-res.com/articles/suppl/m389 p139_app.xls).

\section{Trematode richness and infection levels}

In all samples for which total trematode inventories were available, most crustacean populations contained only 1 trematode species (Fig. 1). Only a single host population was infected with the maximum of 6 trematode species (Fig. 1). The prevalence of single parasite species was $<10 \%$ in most host populations, and the majority of parasite species showed mean intensities and mean abundance of $<5$ parasites per host individual (Fig. 1). Across all individual parasite species samples, mean intensity was positively correlated with prevalence $\left(R^{2}=0.33, p<0.001\right)$, i.e. parasite species occurring at high prevalence also tended to show high intensities (Fig. 2).

\section{Differences among crustacean groups}

The mean number of trematode species (log-transformed) found in a host population varied among host groups with at least 2 entries (ANCOVA; $F_{6,149}=12.8$, $\mathrm{p}<0.001$ ). While amphipod, crab and isopod hosts harboured between 2 and 2.5 trematode species, all other taxa harboured only half as many species (1 to 1.2 species) (Fig. 3). Prevalence, mean intensity and mean abundance also differed among host groups, with at least 3 entries (ANCOVA - prevalence: $F_{6,223}=3.6, \mathrm{p}=$ 0.002; intensity: $F_{6,109}=5.0, \mathrm{p}<0.001$; abundance: $\left.F_{6,127}=5.5, \mathrm{p}<0.001\right)$. While differences among host
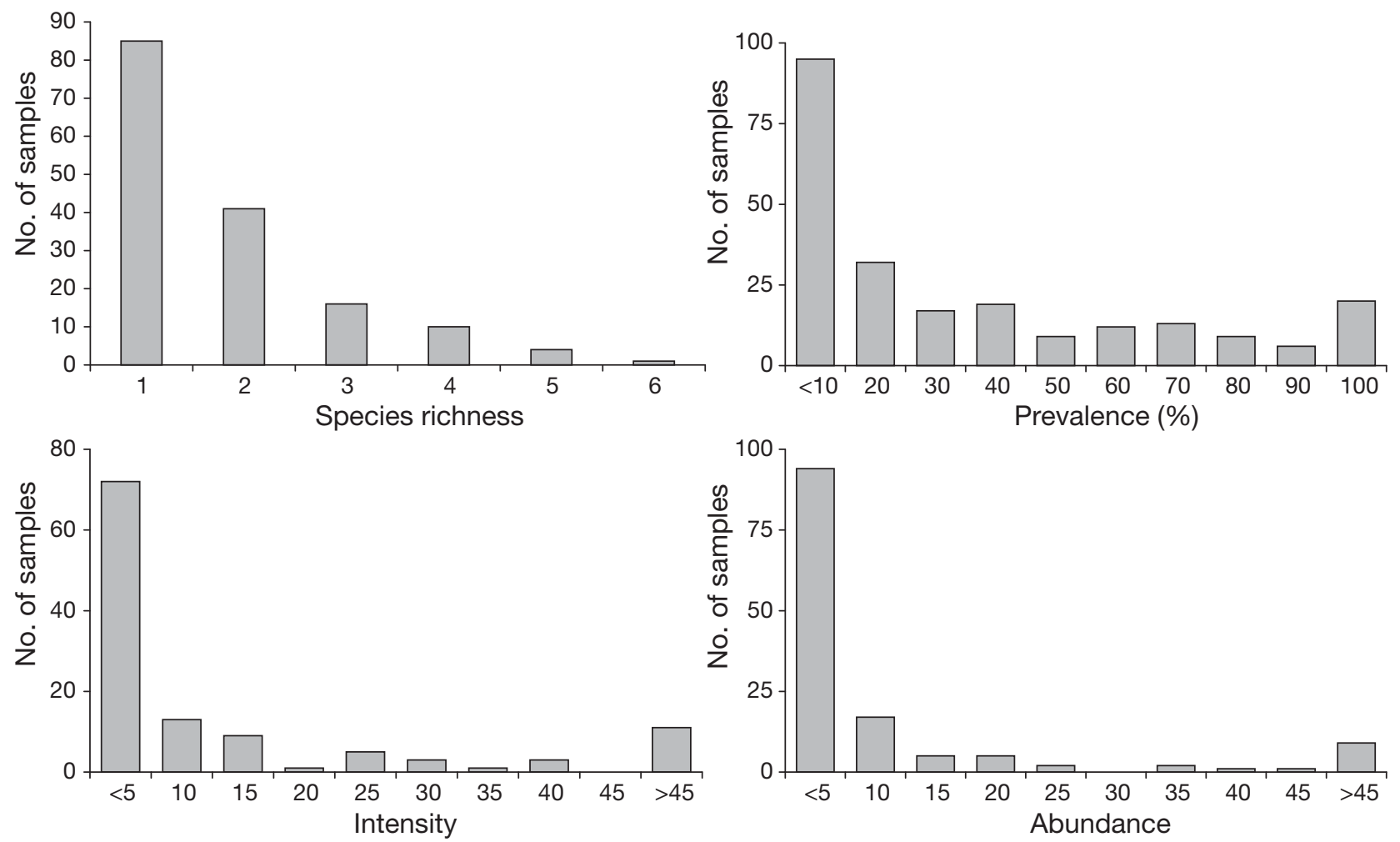

Fig. 1. Frequency distributions of individual trematode species richness, prevalence, mean intensity and mean abundance in marine crustacean hosts among all samples in the data set $(n=157$ samples for trematode richness; 232 for prevalence; 118 for intensity; 62 for abundance) 
groups were less marked for prevalence, ranging from 25 to $50 \%$, intensity and abundance differed more strongly, with brachyuran crabs showing by far the highest values (Fig. 3). There was no effect of sampling effort on the number of trematode species, prevalence, intensity and abundance in the ANCOVAs.

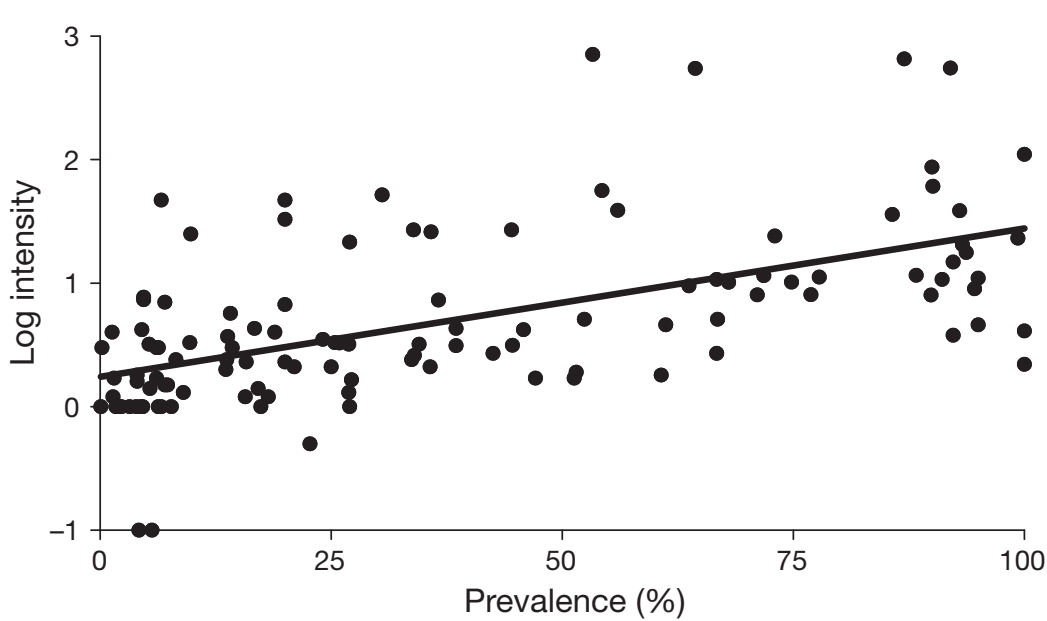

Fig. 2. Relationship between prevalence (\% hosts infected) and log mean intensity of metacercarial infections of marine crustaceans across all samples in the data set $(n=118)$. The line represents the best fit of a regression $(y=0.012 x+0.241)$

\section{Latitudinal gradients in amphipod hosts}

Trematode richness, prevalence, mean intensity and mean abundance all declined with increasing latitude in amphipod hosts (Fig. 4). Latitude was a significant predictor in all 4 GLMs, when controlling for the potentially confounding variables sampling effort, host size and host genus (Table 1). The GLM indicated that sampling effort had no effect on trematode richness or infection level patterns (Table 1). In contrast host size and host genus were significant variables in the GLM on mean intensity and mean abundance of trematodes in amphipods (Table 1). However, these 2 variables were not independent from each other as the genera differed in host size in both the intensity $\left(F_{2,45}=4.67, \mathrm{p}=0.014\right)$ and abundance $\left(F_{2,45}=4.66, \mathrm{p}=0.014\right)$ data sets. Hence, the significant effect of host genus was probably due to differences in host size and not phylogenetic factors per se.
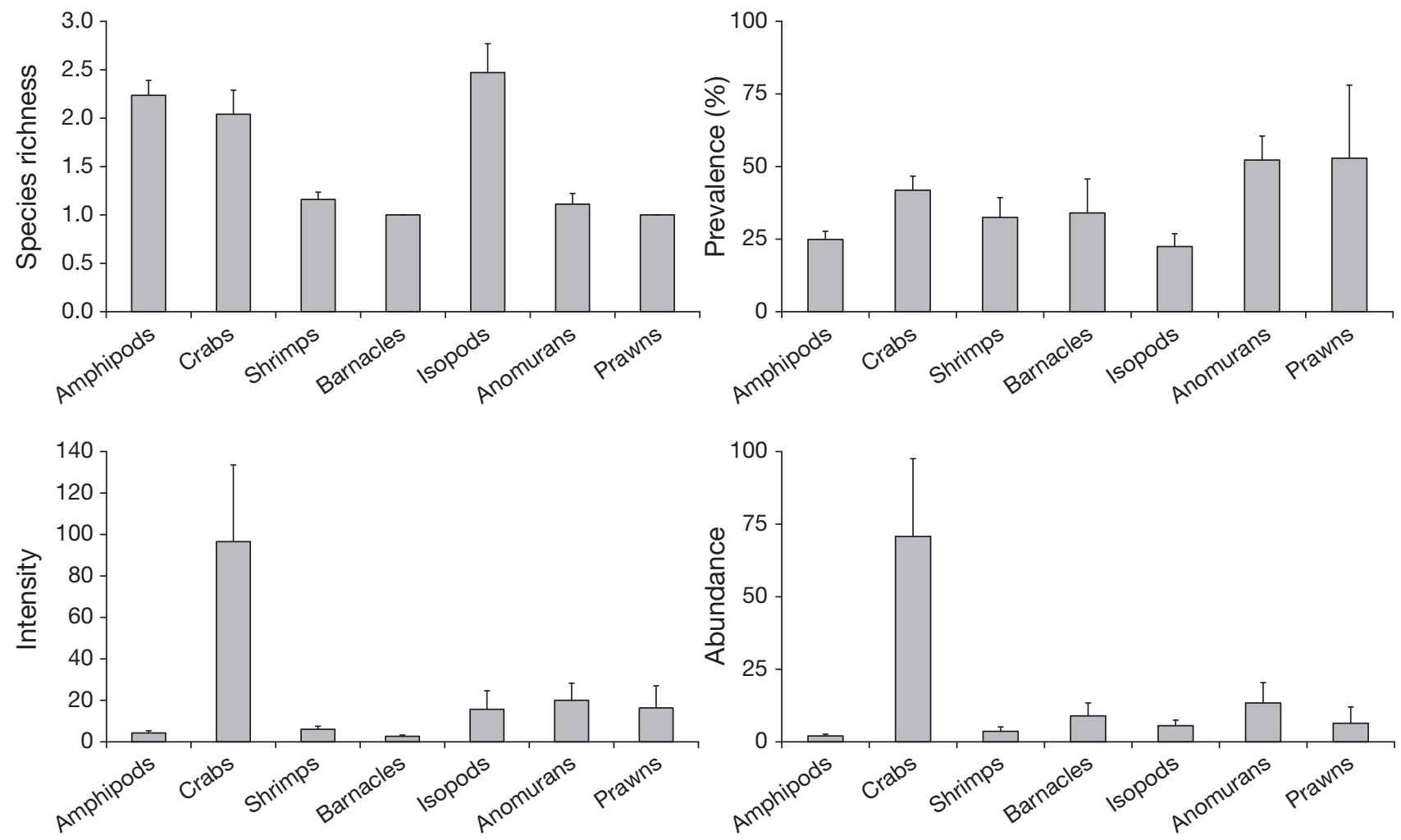

Fig. 3. Differences in mean individual trematode species richness, prevalence, mean intensity and mean abundance (+SE) among different crustacean host groups. Graphs show non-transformed data. Number of respective entries for amphipods, crabs, shrimps, barnacles, isopods, anomurans and prawns: richness: 55, 25, 25, 24, 17, 9, 2; prevalence: 102, 46, 19, 7, 43, 11, 3; intensity: 48, 31, 16, 6, 3, 11, 2; abundance: 48, 32, 16, 23, 3, 11, 2 

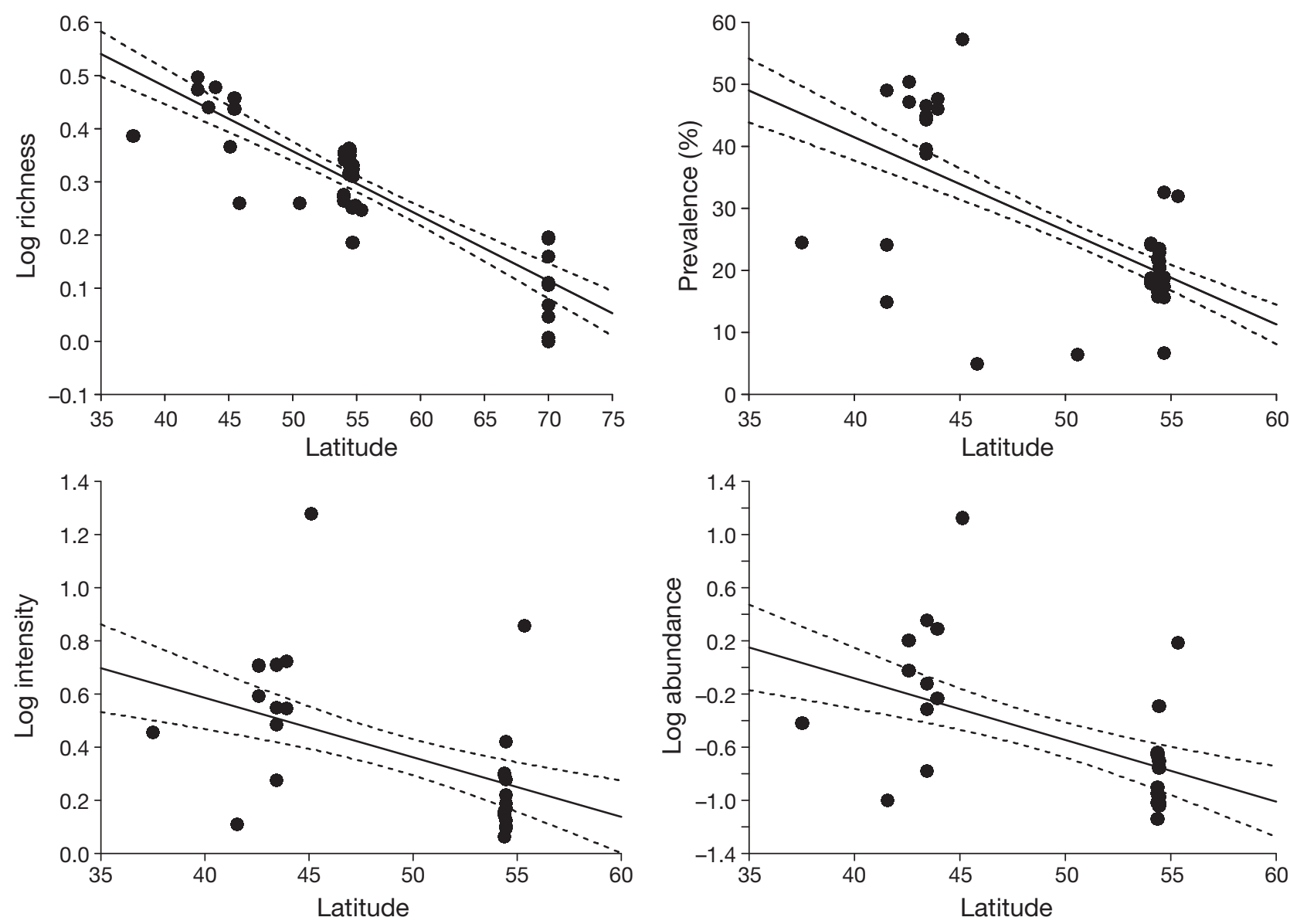

Fig. 4. Latitudinal gradients in individual trematode species richness, prevalence, mean intensity and mean abundance in metacercarial infections of marine amphipod hosts ( $\mathrm{n}=55$ entries for trematode richness; 102 for prevalence; 48 for intensity; 48 for abundance). The graphs show the predicted values from the 4 general linear models, correcting for sampling effort, host size and host genera. The lines represent the best fit of linear regressions through the predicted values plus the $95 \%$ confidence intervals

\section{DISCUSSION}

\section{Low trematode richness and moderate infection levels}

The analyses revealed that crustacean hosts overall harbour a limited richness of trematode parasite fauna, with most host populations being infected by only 1 or 2 trematode species (Fig. 1). The low trematode richness in host populations may be the result of a relatively high host specificity of the trematodes, allowing only few trematode species to infect a specific host species. However, host specificity of trematodes in second intermediate hosts is generally considered to be very low (Galaktionov \& Dobrovolskij 2003), and studies on trematode infections in sympatric host species suggest that many trematode species infect multiple crustacean hosts (e.g. Zander et al. 2002). An alternative explanation for the low trematode richness might thus be that there are only a limited number of trematode species in a given system that fill the niche of crus- tacean parasites. The first intermediate gastropod hosts usually carry a wide range of trematode species that use a variety of second intermediate hosts from many different taxa (Lauckner 1980, Galaktionov \& Dobrovolskij 2003), including other gastropods (sometimes gastropods serve as second intermediate hosts too), bivalves, fish, polychaetes, or even no host (i.e. hard substrates in the environment). Hence, trematodes use an array of transmission pathways, and the low trematode richness in the crustacean hosts in our data set suggests that crustaceans are only used by a small subset of the trematode fauna in a given system. However, our knowledge on host specificity, as well as on the abundance and distribution of trematodes in crustacean hosts in different systems, is very limited. Further studies are needed to clarify the underlying mechanisms.

Whatever the exact mechanisms, the low trematode richness in crustacean hosts indicates that most host populations carry moderate burdens in terms of the number of parasite species. Similarly, infection levels 
Table 1. Results of general linear model testing for the effect of latitude on trematode richness and infection levels (prevalence, mean intensity, mean abundance) in marine amphipod hosts, controlling for sampling effort, maximum host size and host genus

\begin{tabular}{|lcccc|}
\hline & df & MS & $F$ & $p$ \\
\hline Richness & & & & \\
Effort & 1 & 0.003 & 0.074 & 0.786 \\
Size & 1 & 0.016 & 0.402 & 0.529 \\
Genus & 2 & 0.040 & 1.025 & 0.366 \\
Latitude & 1 & 0.325 & 8.246 & 0.006 \\
Error & 49 & 0.039 & & \\
Prevalence & & & & \\
Effort & 1 & 119.985 & 0.168 & 0.683 \\
Size & 1 & 917.11 & 1.286 & 0.26 \\
Genus & 4 & 1243.756 & 1.744 & 0.147 \\
Latitude & 1 & 8593.334 & 12.050 & $<0.001$ \\
Error & 94 & 713.1079 & & \\
Intensity & & & & \\
Effort & 1 & 0.196 & 2.266 & 0.14 \\
Size & 1 & 0.785 & 9.06 & 0.004 \\
Genus & 2 & 0.954 & 11.012 & $<0.001$ \\
Latitude & 1 & 0.953 & 11.001 & 0.002 \\
Error & 42 & 0.087 & & \\
Abundance & & & & \\
Effort & 1 & 1.714 & 2.98 & 0.092 \\
Size & 1 & 4.334 & 7.535 & 0.009 \\
Genus & 2 & 3.881 & 6.747 & 0.003 \\
Latitude & 1 & 5.642 & 9.809 & 0.003 \\
Error & 42 & 0.575 & & \\
\hline
\end{tabular}

by single parasite species were also relatively moderate in most cases (Fig. 1). Most trematode species infect $<20 \%$ of the individuals in their host populations, and almost two-thirds of the parasite species occur with a mean intensity or abundance of $<5$ individual parasites per host. As the effects of trematodes on their crustacean hosts (e.g. mortality) are generally dependent on the density of metacercariae (Fredensborg et al. 2004), the observed pattern suggests that effects of trematodes on crustacean hosts are probably moderate in most cases. However, there are parasite species that exhibit high infection levels in some hosts, both in terms of prevalence and mean intensity/abundance (Fig. 1). In addition, intensity increases with prevalence (Fig. 2), suggesting that host populations in which a specific parasite species is common also experience higher levels of parasite burden. The resulting high parasite burdens might be specific for some host-parasite interactions, i.e. some parasite species exert consistently high costs on their host species, while others are always less severe. Indeed, there appears to be significant repeatability of infection levels within certain host-parasite species combinations throughout their geographical ranges (Thieltges et al. 2009b). Another, but not mutually exclusive, explana- tion for the varying magnitudes of infection levels reported here might be temporal aspects of infection patterns. It is well known that infection levels fluctuate in a local host population across seasons. For example, extraordinarily warm spring temperatures can cause a massive release of cercarial transmission stages from snails that then proceed to infect crustacean hosts, resulting in subsequent high infection levels and even mass mortality events (Mouritsen \& Jensen 1997, Mouritsen et al. 1997). Hence, under certain environmental conditions, infection levels and the concomitant effects on crustacean hosts can be substantial. The data in our analyses do not mirror these temporarily high infection levels as we used mean values for each host-parasite species pair at a certain location. Hence, the potential density-dependent effects of trematodes on their host populations due to high infection levels may actually be more common than our analyses suggest.

\section{Different patterns among crustacean groups}

As evident from Fig. 3, not all host groups were similarly infected by trematodes. In amphipods, crabs and isopods, trematode richness was twice as high as in other groups, and crabs in particular showed very high mean intensity and abundance. The latter observation can probably be explained by the larger size of crabs compared to most other groups. Infection levels of parasites are usually positively correlated with the size (and longevity) of their hosts (Poulin 2007). The same applies to parasite richness patterns, with larger hosts usually harbouring more parasite species than smaller hosts (Poulin 2007). In contrast to crabs, host size cannot explain why amphipod and isopod host populations harbour similarly high trematode species richness, as both these groups are usually quite small (5 to $30 \mathrm{~mm}$ in our data set, see Table S1). It might be that the high abundance of both groups in marine benthic systems makes them particularly suitable hosts for trematodes. We must point out that the patterns observed might be skewed by the low sample sizes in some of the host groups. More parasite inventories of these groups (especially from anomurans, prawns and isopods) are needed to validate the observed patterns.

\section{Latitudinal gradient of parasitism in amphipod hosts}

Latitude had a significant effect on trematode richness, as well as infection levels, in amphipods: all measures of parasitism decreased with increasing latitude (Fig. 4, Table 1). The observed decrease of prevalence, mean intensity and mean abundance with increasing 
latitude may be related to a general temperature gradient from higher to lower water temperatures, as latitude is a proxy for temperature. Generally higher water temperatures at low latitudes can be expected to result in a higher supply of infective cercarial stages as the production of cercariae inside snails is strongly temperature dependent (Poulin 2006). In return, the higher supply of cercariae can be expected to result in increased infection levels in the second intermediate hosts. The potential importance of trematode parasites for the population ecology of crustaceans can therefore be dependent on latitude: as one goes from $55^{\circ}$ to $40^{\circ}$ latitude, both the mean prevalence and mean intensity of trematode infections show a 2-fold increase (see Fig. 4). However, the relationship between latitude and infection levels is not a perfectly linear one, as there is considerable scatter in the data and many data points fall outside the $95 \%$ confidence interval (Fig. 4). This might be an artefact of the data set resulting from the temporal variability discussed above. Furthermore, the scatter might also result from varying environmental conditions modifying the general effect of temperature and latitude. The transmission of cercariae from the first to the second intermediate hosts is a complex interplay of abiotic and biotic factors. For example, habitat characteristics can affect cercarial transmission (Thieltges \& Reise 2007), and non-host organisms like filter feeders and predators can also interfere with the transmission of cercariae (Thieltges et al. 2008). These factors will almost certainly vary among localities at the same or different latitudes and are likely to cause considerable heterogeneity in infection levels. Considering this small-scale heterogeneity, it is noteworthy that we could nevertheless detect an effect of latitude on infection levels. This suggests that latitude is a relatively strong determinant of infection levels in second intermediate amphipod hosts. However, the number of locations available from various latitudes is limited in our data set, and the data are clearly concentrated between $40^{\circ}$ and $45^{\circ}$ as well as around $55^{\circ}$ (Fig. 4). In addition, the range of latitudes from which data are available is relatively narrow, including neither locations close to the poles nor to the equator and including only a few data from the southern hemisphere. All this certainly limits the power of our analyses, and more parasite inventories, particularly from low and high latitudes, are needed to validate our findings.

Compared with data on prevalence, mean intensity and mean abundance, the decline in trematode species richness with increasing latitude is more convincing, since the available data span over a larger range of latitudes (Fig. 4). However, the observed pattern is surprising given the (almost) lack of such a pattern in the first intermediate gastropod hosts (Poulin \& Mouritsen 2003). It might be that the number of potential host species is limited at higher latitudes, as the richness of free-living organisms generally decreases with latitude in the marine realm (Hillebrand 2004b). This might decrease the chances for trematode species that preferentially infect a single host species (but can infect other host species to a lesser extent) to become established in a system, thus reducing the total number of crustacean trematodes in this system. It might also be that the strong host specificity exhibited by trematodes in first intermediate hosts confounds any patterns, as the availability of the hosts may be more important than any environmental factors. In contrast, host specificity is much lower in second intermediate hosts, and trematodes may be able to utilise a variety of hosts; thus, environmental factors may be more relevant for this stage in the parasite's life cycle. However, this remains to be studied, and our data are certainly limited by a restricted range of latitudes and considerable scatter in the data (see above).

\section{Conclusions}

Our data indicate that populations of second intermediate crustacean hosts generally harbour a low number of trematode species and exhibit moderate infection levels. This suggests that the density-dependent effects on hosts caused by parasites are limited to a few host-parasite systems or to particular local outbreaks. Crabs, isopods and amphipods harbour twice as many parasite species as other groups, identifying them as preferred transmission pathways of trematodes and also as the host groups most affected by trematode parasitism. Trematode richness as well as infection levels (prevalence, mean intensity, mean abundance) all declined with increasing latitude in amphipod hosts. Although hampered by the lack of data at very low and high latitudes, this finding points to a role of latitude in determining trematode richness and infection levels in second intermediate hosts, probably causally linked to temperature. It is unclear if these results can be transferred to other second intermediate host groups (e.g. bivalves), and future studies will be interesting in this respect.

Acknowledgements. D.W.T. acknowledges support by a fellowship from the German Research Foundation (DFG) (Th 1361/1-1).

\section{LITERATURE CITED}

Attrill MJ, Stafford R, Rowden AA (2001) Latitudinal diversity patterns in estuarine tidal flats: indications of a global cline. Ecography 24:318-324

Fredensborg BL, Mouritsen KN, Poulin R (2004) Intensitydependent mortality of Paracalliope novizealandiae (Am- 
phipoda: Crustacea) infected by a trematode: experimental infections and field observations. J Exp Mar Biol Ecol 311:253-265

Galaktionov KV, Dobrovolskij AA (2003) The biology and evolution of trematodes: an essay on the biology, morphology, life cycles, transmission, and evolution of digenetic Trematodes. Kluwer Academic Publishers, Boston

Gregory RD, Blackburn TM (1991) Parasite prevalence and host sample size. Parasitol Today 7:316-318

Hillebrand H (2004a) On the generality of the latitudinal diversity gradient. Am Nat 163:192-211

Hillebrand H (2004b) Strength, slope and variability of marine latitudinal gradients. Mar Ecol Prog Ser 273:251-267

Lauckner G (1980) Diseases of Mollusca: Gastropoda. In: Kinne O (ed) Diseases of marine animals, Vol I. General aspects, Protozoa to Gastropoda. Wiley, New York, p 311-424

Mittelbach GG, Schemske DW, Cornell HV, Allen AP and others (2007) Evolution and the latitudinal diversity gradient: speciation, extinction and biogeography. Ecol Lett 10:315-331

Mouritsen KN (2002) The Hydrobia ulvae-Maritrema subdolum association: influence of temperature, salinity, light, water-pressure and secondary host exudates on cercarial emergence and longevity. J Helminthol 76: 341-347

Mouritsen KN, Jensen KT (1997) Parasite transmission between soft-bottom invertebrates: temperature mediated infection rates and mortality in Corophium volutator. Mar Ecol Prog Ser 151:123-134

Mouritsen KN, Jensen T, Jensen KT (1997) Parasites on an intertidal Corophium bed: factors determining the phenology of microphallid trematodes in the intermediate host populations of the mudsnail Hydrobia ulvae and the amphipod Corophium volutator. Hydrobiologia 355:61-70

Pianka ER (1966) Latitudinal gradients in species diversity: a review of concepts. Am Nat 100:33-46

Poulin R (2006) Global warming and temperature-mediated increases in cercarial emergence in trematode parasites. Parasitology 132:143-151

Poulin R (2007) Evolutionary ecology of parasites. Princeton University Press, Princeton, NJ

Poulin R, Morand S (2004) Parasite biodiversity. Smithsonian Books, Washington, DC

Poulin R, Mouritsen KN (2003) Large-scale determinants of trematode infections in intertidal gastropods. Mar Ecol Prog Ser 254:187-198

Poulin R, Rohde K (1997) Comparing the richness of metazoan ectoparasite communities of marine fishes: controlling for host phylogeny. Oecologia 110:278-283

Rohde K (1992) Latitudinal gradients in species diversity: the

Editorial responsibility: Lisandro Benedetti-Cecchi, Pisa, Italy search for the primary cause. Oikos 65:514-527

Rohde K (1993) Ecology of marine parasites, 2nd edn. CAB International, Wallingford

Rohde K (1998) Latitudinal gradients in species diversityArea matters, but how much? Oikos 82:184-190

Rohde K (1999) Latitudinal gradients in species diversity and Rapoport's rule revisited: a review of recent work and what can parasites teach us about the causes of the gradients? Ecography 22:593-613

Rohde K (2002) Ecology and biogeography of marine parasites. Adv Mar Biol 43:1-86

Rohde K, Heap M (1998) Latitudinal differences in species and community richness and in community structure of metazoan endo- and ectoparasites of marine teleost fish. Int J Parasitol 28:461-474

Schmidt-Nielsen K (1997) Animal physiology: adaptation and environment, 5th edn. Cambridge University Press, Cambridge

Thieltges DW (2006) Effect of metacercarial trematode infections (Renicola roscovita) on growth in intertidal blue mussels (Mytilus edulis). Mar Ecol Prog Ser 319:129-134

Thieltges DW, Reise K (2007) Spatial heterogeneity in parasite infections at different scales in an intertidal bivalve. Oecologia 150:569-581

> Thieltges DW, Rick J (2006) Effect of temperature on emergence, survival and infectivity of cercariae of the marine trematode Renicola roscovita (Digenea: Renicolidae). Dis Aquat Org 73:63-68

> Thieltges DW, Jensen KT, Poulin R (2008) The role of biotic factors in the transmission of free-living endohelminth stages. Parasitology 135:407-426

Thieltges DW, Ferguson MAD, Jones CS, Noble LR, Poulin R (2009a) Biogeographical patterns of marine larval trematode parasites in two intermediate snail hosts in Europe. J Biogeogr 36:1493-1501

> Thieltges DW, Fredensborg BL, Poulin R (2009b) Geographical variation in metacercarial infection levels in marine invertebrate hosts-parasite species character versus local factors. Mar Biol 156:983-990

Walther BA, Cotgreave P, Price RD, Gregory RD, Clayton DH (1995) Sampling effort and parasite species richness. Parasitol Today 11:306-310

Willig MR, Kaufman DM, Stevens RD (2003) Latitudinal gradients of biodiversity: pattern, process, scale, and synthesis. Annu Rev Ecol Syst 34:273-309

Willmer P, Stone G, Johnston I (2000) Environmental physiology of animals. Blackwell Science, Oxford

Zander CD, Koçuglo Ö, Skroblies M, Strohbach U (2002) Parasite populations and communities from the shallow littoral of the Orther Bight (Fehmarn, SW Baltic Sea). Parasitol Res 88:734-744

Submitted: January 5, 2009; Accepted: June 26, 2009

Proofs received from author(s): August 26, 2009 